\title{
NEW METHOD OF REFLECTOR SURFACE SHAPING TO PRODUCE A PRESCRIBED CONTOUR BEAM ${ }^{1}$
}

\author{
Boris V. Semenov ${ }^{1,2, \dagger}$, Nikolai I. Chernykh ${ }^{1,2, \dagger \dagger}$, Viktor M. Pleshchev ${ }^{1, \dagger \dagger \dagger}$ \\ ${ }^{1}$ Krasovskii Institute of Mathematics and Mechanics, \\ Ural Branch of the Russian Academy of Sciences, Ekaterinburg, Russia; \\ ${ }^{2}$ Ural Federal University, Ekaterinburg, Russia, \\ †b.v.semenov@urfu.ru, ${ }^{\dagger}$ chernykh@imm.uran.ru, ${ }^{\dagger \dagger}$ Viktor.Pleshchev@imm.uran.ru
}

\begin{abstract}
In this paper a simple iterative synthesis method is presented for the formation of the shape of the reflector surface with a single feed element to produce the desired contour beam. This is the method of the optimal phase synthesis of the appropriate field in the reflector aperture similar to other works. But unlike them, we solve the problem in a very simple way using the properties of complex-valued functions and Fourier transforms and not applying complicated methods of numerical minimization theory.
\end{abstract}

Key words: Antenna, Shaped reflector, Radiation pattern, Contour beam, Synthesis.

\section{Introduction}

We consider the problem of synthesis of antenna reflector surface with a single feed. Such surfaces are constructed to generate a desirable far-field pattern available for the reflector aperture.

There are antennas with a single feed that form contour beams by means of appropriate profiled reflector surfaces for serving separated districts from spacecraft. They have a very simple construction, are reliable in exploitation, and optimally solve problems of electromagnetic compatibility.

Several synthesizing methods are known for the problem. They proved themselves to be efficient but are related to the minimization problems of multi-parameter goal functions. Some of these methods are direct methods for optimal modification of the basis reflector surface represented by polynomials, splines, and wavelets [1-4]. Other methods are related to preliminary synthesizing the electromagnetic fields in the reflector aperture which generate the assembly of narrow partial beams with subsequent optimization of the disposition of their maximums and selection of their superposition parameters. After that, the computation of the reflector surface form is carried out to generate the synthesized optimal aperture field [5-10]. The developed methods are very efficient for the synthesis of the reflector surface with the diameter of several tenth of the wavelength.

In this paper, another method is presented for contour beam synthesizing by antenna with a single feed element and a special reflector shape. It is also related to solving optimization problems but without the application of multi-parameter nonlinear optimization theory. The method is entirely based on the specific setting of the reflector surface synthesizing problem and on the properties of complex-valued functions and Fourier transforms. It is possible to adapt the method to the problem of phase control of large radiating arrays in real time.

The computation of the electric field in opening of the initial reflector (for example, parabolic revolution with focus at the phase center of the feeding element) is carried out in the knots of the uniform lattice at very close aperture. It is implemented by means of a vector radiation model of

\footnotetext{
${ }^{1}$ This work was supported by the Program for State Support of Leading Scientific Schools of the Russian Federation (project no. NSh-9356.2016.1) and by RAS Presidium programm "Mathematical Problems of Modern Control Theory".
} 
the array, which has a sufficiently dense set of points on the initial reflector surface. Dimensions of the lattice cells must guarantee the exact calculation of the Kirchhoff integral for the far zone field.

Our method, like many others, is iterative. The phase synthesis of a given pattern for the minimization of the amplitude distribution deviation from the desired far-field pattern is performed in $L^{2}\left(\mathbb{R}^{2}\right)$ space. We apply (here, perhaps, for the first time) the iterative methods alternately using direct and inverse Fourier transforms. It does not require any classic ways of numerical differentiation and solutions of large systems of linear equations. The mentioned iteration procedure is implemented until a completely suitable phase distribution at the reflector aperture is found. Moreover, the procedure may be accompanied by a proper shaping of the reflector surface at every step of the iteration. To represent intermediate variants of the constructed surface, we make it "scaly". Every "scale" is a fragment of a parabolic surface having the same focus as the initial one. Of course, we do not consider the diffraction at the edges of these (virtual) "scales", because, at the final stage of optimization, the weakly discontinuous surface will be changed by a continuous and smooth one.

\section{Physical and phenomenological basis of the method}

Bellow, the size and contour of the aperture, the pattern of the feed element, and model $D(\theta, \varphi)$ of the required far-field radiation pattern are supposed to be given. The latter can be a model of different beams, including contour ones, whose parameters conform to the reflector opening.

Getting to the problem, we assume that the initial reflector surface is cut off from a paraboloid of revolution by a plane orthogonal to its axis. The corresponding part of the plane is assumed to be its aperture $A$ containing the origin $O$ and the axes $O X$ and $O Y$ of the Cartesian coordinate system, whose axis $O Z$ coincides with the axis of the parabolic surface and contains its focus $F$ coincident with the phase center of the feed element.

At first, it is necessary to the calculate electric field at the aperture $A$ (more exactly, its component $\dot{E}(x, y)$ on the chosen polarization). It should be done very precisely, because it is possible (although not necessary) do not change the amplitude distribution $E(x, y)=|\dot{E}(x, y)|$ in the aperture $A$ in future (neglecting the weak influence of local shifts of primary reflector surface cells). The phase $S_{0}(x, y)=\arg \dot{E}(x, y) /(2 \pi)$ will be the initial functional "parameter" which we are going to change in the course of the reflector surface synthesis. The electrodynamic problem of searching the field $\dot{E}(x, y)$ is not considered in this article, since it can be solved by other known methods. We found that the computation according to the vector model formulas (see, for example, [13]) is very efficient.

\section{Model of the algorithm}

First we expound a continuous version of the algorithm for the synthesis of the reflector surface, which will be necessary to carry out numerically. Knowing $\dot{E}(x, y)$ in the reflector aperture $A$, we have a representation of the electric field expected in the far zone as the Kirchhoff integral

$$
\widehat{E}(u, v)=\iint_{A} \dot{E}(x, y) \mathrm{e}^{-2 \pi \mathrm{i}(u x+v y)} d x d y,
$$

where $u=k \sin \theta \cos \varphi, v=k \sin \theta \sin \varphi,(\theta, \varphi)$ are the angles of the spherical coordinate system, $k=1 / \lambda$, and $u, v$ are dimensionless variables, because $x, y$ are measured in wavelengths. The function $S_{0}(x, y)$ is determined from the condition $\dot{E}(x, y)=E(x, y) \exp \left(2 \pi i S_{0}(x, y)\right)$. Although it involves the phase component of the feed, it can be interpreted as the length of the optical path from a point $(x, y) \in A$ through the corresponding point on the reflector surface and then up to 
the phase centre of the feed (also measured in wavelength). Disregarding diffraction at the edge of the reflector, we suppose that the antenna radiation extends only to the half-space $z>0$ and $E(x, y) \equiv 0$ under the condition $(x, y) \in R^{2} \backslash A$. Thus, from the previous reasoning and (2.1), we have

$$
\widehat{E}(u, v)=\widehat{E}(u, v, S)=\iint_{\mathbb{R}^{2}} E(x, y) e^{2 \pi i S(x, y)} e^{-2 \pi i(u x+v y)} d x d y, \quad S=S_{0},
$$

that is the Fourier transform of the predetermined function $\dot{E}(x, y)$. If needed, we smoothly extend the given contour model $D(u, v)$ of the radiation pattern a little outside the domain of interest and, denoting the new domain by $\Omega$, we set $D(u, v) \equiv 0$ for $(u, v) \in \mathbb{R}^{2} \backslash \Omega$. We can define the mean square deviation by the formula

$$
\Delta(D, \widehat{E})=:\|D(u, v)-|\widehat{E}(u, v ; S)|\|=\left(\iint_{\mathbb{R}^{2}}(D(u, v)-|\widehat{E}(u, v ; S)|)^{2} d u d v\right)^{1 / 2}
$$

and formulate the following problem of antenna phase synthesis: to find a function $S(x, y)=$ $S(x, y ; D)$ for which the value

$$
\delta=: \inf _{S} \Delta(D(u, v), \widehat{E}(u, v ; S))
$$

is attained. Here, $S$ belongs to the class of all measurable real-valued functions. It should be noted that only a part of $R^{2}$ (the ball $u^{2}+v^{2} \leq 1$ ) really lies in the physical space. But, in problem (2.4), we also minimize the energy flow

$$
\iint_{u^{2}+v^{2}>1}|\widehat{E}(u, v ; S)|^{2} d u d v=\iint_{u^{2}+v^{2}>1}|D(u, v)-\widehat{E}(u, v ; S)|^{2} d u d v
$$

in the antenna reactive zone. Further, we apply the usual iterative procedures for phase synthesis worked out for hybrid reflector antennas (HRA) (see, for example, [11, 12]), but here they are especially clear because do not use a finite-dimensional approximation of the antenna radiation $\widehat{E}(u, v ; S)$.

Obviously, we have

$$
\begin{aligned}
\delta^{2} & =\inf _{S} \inf _{\psi} \iint_{\mathbb{R}^{2}}\left|D(u, v) e^{i \psi(u, v)}-F^{-}\left(E(x, y) e^{2 \pi i S(x, y)}\right)(u, v)\right|^{2} d u d v= \\
& =\inf _{\psi} \inf _{S} \iint_{\mathbb{R}^{2}}\left|F^{+}\left(D(u, v) e^{i \psi(u, v)}\right)(x, y)-E(x, y) e^{2 \pi i S(x, y)}\right|^{2} d x d y,
\end{aligned}
$$

where $\psi(u, v)$ is a real-valued measurable function like $S(x, y)$ and functions $\left(F^{ \pm} g\right)(s, t)$ are inverse $($ with +$)$ and direct $($ with -$)$ Fourier transforms defined for a function $g \in L\left(\mathbb{R}^{2}\right)$ by

$$
\left(F^{ \pm} g\right)(s, t)=\iint_{\mathbb{R}^{2}} g(\xi, \zeta) e^{ \pm 2 \pi i(s \xi+t \zeta)} d \xi d \zeta
$$

and then extended to $L^{2}\left(\mathbb{R}^{2}\right)$ in a reasonable well-known way. To represent $\delta$ defined by (2.4) as like (2.5), we have used the following considerations:

1) from the properties of complex-valued functions, it follows that the first integrand in (2.5) is minimal for $\psi$ equal to $\psi_{\mathrm{s}}$ defined

$$
\left.\psi_{S}(u, v)=: \arg \widehat{E}(u, v ; S)=\arg \left(F^{-}\left(E \mathrm{e}^{2 \pi \mathrm{i} S}\right)\right)(u, v)=\arg F^{-}(\dot{E})\right),
$$


so the inner infimum over $\psi$ coincides with $\Delta^{2}(D, \dot{E})$ (see $(2.3)$ ) in view of formulas $(2.2)-(2.4)$ and the definition of $F^{-} g$;

2 ) it is easy to see that, for any positive functional $G(S, \psi)$ on the spaces of real-valued measurable functions $S(x, y)$ and $\psi(u, v)$, the following formula $\inf _{S} \inf _{\psi} G(S, \psi)=\inf _{\psi} \inf _{S} G(S, \psi)$ holds;

3) the equality of integrals in (2.5) follows from the Parseval equality.

Reasoning as in 1), we see that the inner infimum in the last part of $(2.5)$ is attained for $2 \pi S$ equal to

$$
2 \pi S(x, y)=2 \pi S_{\psi}(u, v)=: \arg \left(F^{+}\left(D e^{i \psi}\right)\right)(x, y) .
$$

From these considerations, it follows that the solution $S(x, y)((x, y) \in A)$ of problem (2.4) together with the solution $\psi$ of the problem

$$
\inf _{\psi} \iint_{\mathbb{R}^{2}}\left(E(x, y)-\left|F^{+}\left(D e^{i \psi}\right)(x, y)\right|\right)^{2} d x d y
$$

must be connected by the nonlinear equations (2.6) and (2.7). Except for special cases, this system can be solved only approximately by a numerical method.

To construct such a method, we use the obvious fact that, for every above mentioned functions $S(x, y)$ and $\psi(u, v)$, we have the following inequalities for the norms in the space $L^{2}\left(\mathbb{R}^{2}\right)$ hold:

$$
\begin{gathered}
\left\|D e^{i \psi_{s}}-F^{-}\left(E e^{2 \pi i S}\right)\right\| \leq\left\|D e^{i \psi}-F^{-}\left(E e^{2 \pi i S}\right)\right\| \\
=\left\|F^{+}\left(D e^{i \psi}\right)-E e^{2 \pi i S}\right\|, \\
\left\|F^{+}\left(D e^{i \psi}\right)-E e^{2 \pi i S_{\psi}}\right\| \leq\left\|F^{+}\left(D e^{i \psi}\right)-E e^{2 \pi i S}\right\|_{L^{2}(R)},
\end{gathered}
$$

where $\psi_{S}$ and $S_{\psi}$ are defined in (2.6) and (2.7). Further, beginning with the function $S_{0}(x, y)$ and alternately using formulas (2.6) and (2.7), we construct the following chain of functions:

$$
\begin{gathered}
\psi_{S_{0}}(u, v), S_{\psi_{S_{0}}}(x, y)=: S_{1}(x, y), \psi_{S_{1}}(u, v), \\
S_{2}(x, y)=: S_{\psi_{S_{1}}}(x, y), \ldots, \psi_{S_{n-1}}(u, v), S_{n}=: S_{\psi_{S_{n-1}}}, \ldots
\end{gathered}
$$

Denoting

$$
\left\{\begin{array}{l}
S_{n}(x, y)-S_{n-1}(x, y)=: \Delta_{n-1}(x, y) \quad(n \in \mathbb{N},(x, y) \in A), \\
\left.\delta^{n}=\left\|D e^{i \psi_{S_{n}}}-F^{-}\left(E e^{2 \pi i S_{n}}\right)\right\|=\| F^{+}\left(D e^{i \psi_{S_{n}}}\right)-E e^{2 \pi i S_{n}}\right) \|, \\
\delta_{n}=\left\|F^{+}\left(D e^{i \psi_{S_{n}}}\right)-E e^{2 \pi i S_{n+1}}\right\|=\| D e^{i \psi_{S_{n}}-F^{-}\left(E e^{2 \pi i S_{n+1}}\right) \|}
\end{array}\right.
$$

and assuming that $S=S_{n}(x, y)$ and $\psi=\psi_{S_{n-1}}(u, v)$ in $(2.8)$ and $S=S_{n}(x, y)$ and $\psi=\psi_{S_{n}}(u, v)$ in $(2.9)$, we deduce the inequalities

$$
\cdots \geq \delta_{n-1} \geq \delta^{n} \geq \delta_{n} \geq \cdots \quad(n=1,2, \ldots) .
$$

Since the formulas $\psi_{S_{n}}=\arg F^{-}\left(E e^{2 \pi i S_{n}}\right)$ and $F^{-}\left(E e^{2 \pi i S_{n}}\right)=\widehat{E}\left(u, v ; S_{n}\right)$ hold, we have that $\delta^{n}=\left\|D-\left|\widehat{E}\left(u, v ; S_{n}\right)\right|\right\|$ is the distance between the desired radiation pattern and the realizable pattern $\left|\widehat{E}\left(u, v ; S_{n}\right)\right|$. This distance decreases to some value $\delta_{\infty}\left(S_{0}\right)$ as $n \rightarrow \infty$, which cannot be zero, because the finitely supported function $D(u, v)$ is not an entire function. For different $S_{0}(x, y)$, the sequences $\left\{S_{n}(x, y)\right\}$ may differ too, since problem (2.4) is a set-valued extremal one (for example, $|\widehat{E}(u, v ; S)| \equiv \mid \widehat{E}(u, v ; S+$ const $) \mid)$. Nevertheless, for large $n$, the function $\left|\widehat{E}\left(u, v ; S_{n}\right)\right|$ inherits the main features of $D(u, v)$. We verified this fact in many computing experiments. 


\section{Computation procedures}

It is natural to begin the construction of the sequence $\left\{S_{n}(x, y)\right\}$ with calculating $S_{0}(x, y)=$ $\arg \dot{E}(x, y)=\dot{E}(x, y) /|\dot{E}(x, y)|$ over the nodes of a chosen lattice and stop it when the replacement of $\psi_{S_{n-1}}$ by $\psi_{S_{n}}$ negligibly changes the value $\max _{A}\left|\Delta_{n-1}(x, y)\right|$ or the value

$$
\max _{\Omega}\left|\widehat{E}\left(u, v ; S_{n-1}\right)-\widehat{E}\left(u, v ; S_{n}\right)\right| .
$$

In practice, the range of values $(u x+v y)$ is not large, so oscillations of the function $\exp (-2 \pi i(u x+$ $v y)$ ) are also not large. Therefore, an approximate computation of the integral in the Fourier transforms over any dense lattice does not cause any difficulties and the construction of the sequence $\left\{S_{n}\right\}$ almost does not need any additional calculations.

\section{Synthesis of the reflector surface}

The initial parabolic reflector surface $z=\frac{x^{2}+y^{2}}{4 f}-z_{0}\left(z_{0}>0, x^{2}+y^{2}<4 f z_{0}\right)$ can be corrected after each step of replacement of $S_{n-1}(x, y)$ by $S_{n}(x, y)$. It is possible to do this more rarely or at the end, i.e., immediately after the computation of $S_{N}$ based on the difference $S_{n}(x, y)-S_{0}(x, y)$. Below, we use the first strategy.

Let cells of the chosen lattice over the aperture $A$ be the squares with size $h \times h$ and midpoints $M_{i j}$ with coordinates $x_{i}=i h, y_{j}=j h(i, j=0, \pm 1, \ldots, \pm k$, where $(k+1 / 2) h=: r$, and $r$ is the radius of $A$ ) and with the conditions $\left(x_{i}, y_{j}\right) \in A$. Here, we have $r=\sqrt{4 f z_{0}}, F\left(0,0, f,-z_{0}\right)$ is the focus of the paraboloid, the cells $m_{i j}$ of the lattice are framed by the lines $\left(x=x_{i} \pm h / 2, z=0\right)$ and $\left(y=y_{j} \pm h / 2, z=0\right)$. Replacing the condition $z=0$ by $z<0$, we obtain, instead of the cells, the set of tubes $t_{i j}$ in the half-space $z<0$ of the space $R^{3}$ whose orthogonal cross-sections are the squares

$$
\left(-\frac{h}{2}+x_{i}<x<x_{i}+\frac{h}{2},-\frac{h}{2}+y_{j}<y<y_{j}+\frac{h}{2}, z=\text { const }<0\right) .
$$

Every tube $t_{i j}$ cuts off an initial scale $\sigma_{i j}^{0}$ from the initial paraboloid which is part of the paraboloid $z=\frac{x^{2}+y^{2}}{4 f}-z_{0},(x, y) \in m_{i j}$, intersecting the axis at $t_{i j}$ at the point $M_{i j}^{0}\left(x_{i}, y_{j}, \frac{x_{i}^{2}+y_{j}^{2}}{4 f}-z_{0}\right)$. Further, we use analogous "scales" which are cuttings of any other paraboloid $P_{i j}$ with the same axis of symmetry $O Z$ and focus $F$. The cuttings are embedded in the tubes $t_{i j}$ and contain any given point $M\left(x_{i}, y_{j}, z_{i j}\right)$ on their axes. It is easy to verify that the equation of the paraboloid $P_{i j}$ for the given $z_{i j}$ is

$$
z=\frac{x^{2}+y^{2}}{4 f_{i j}}-\widetilde{z}_{i j}, \quad(x, y) \in m_{i j}
$$

where the focal distance $f_{i j}$ and the $z$ th vertex coordinate $\widetilde{z}_{i j}$ are defined by the relations

$$
\left\{4 f_{i j}\left(z_{i j}+\widetilde{z}_{i j}\right)=x_{i}^{2}+y_{j}^{2}, f_{i j}-\widetilde{z}_{i j}=f-z_{0}, f_{i j}>0, z_{i j}>0\right\} .
$$

Since the function $\exp (-2 \pi i S(x, y))$ of $S$ is $\lambda$-periodic, it is quite possible to locally change the function $S(x, y)$ in the Kirchhoff integral $(2.2)$ by $(S(x, y)-n)$ with an arbitrary integer $n$ up to $[S(x, y)]$. Hence, taking into account the first notation in (2.10) and determining $S_{1}(x, y)$, we can compensate the difference $\Delta_{1}(x, y)$ by small shifts and simultaneously change all initial scales $\sigma_{i j}^{0}$ by $\sigma_{i j}^{1}$ whose coordinates $z_{i j}^{0}$ are determined from the conditions

$$
\left|F M_{i j}^{0}\right|+\left|M_{i j}^{0} M_{i j}\right|=S_{0}\left(M_{i j}\right)+\Delta_{0}\left(M_{i j}\right)-\Delta_{0}(0,0)+\left(n_{i j}^{0}\right) \lambda .
$$


Here, we assume that $n_{00}^{0}=0$, so, the location of $\sigma_{00}^{0}$ is not changed. The other numbers $n_{i j}^{0}$ determining the points $M_{i j}^{0}$ must be chosen layerwise around $M_{00}\left(0,0,-z_{0}\right)$ to minimize the distance $\left|z_{i j}^{0}-z_{i_{1} j_{1}}^{0}\right|$ between the neighbouring points $M_{i j}$ and $M_{i_{1} j_{1}}$.

The further steps of the iteration procedures are implemented according to the same principles: using (2.6) and (2.7), we find the recurrent phase distribution $S_{0}\left(M_{i j}\right)\left(M_{i j} \in A\right)$ and compensate the differences $\Delta_{n-1}\left(M_{i j}\right)$ by numerical construction of the corresponding new scaled surface of the reflector as explained above in the case $n=1$. Just in this construction, we must replace the set of points $M_{i j}^{0}$ by $M_{i j}^{n}$ and perform all calculations for $n=1,2, \ldots$ according formulas (4.11)-(4.13).

In this section, we described how to choose a number $N$ to stop the iterations. In the final stage, it will be necessary to smooth the constructed weakly discontinuous surface $z=f_{N}(x, y)$ built of little scales $n_{i j}^{N}$. A fairly good result could be obtained as a close solution of the approximation problem

$$
\left\|f_{N}(x, y)-S_{2}(x, y)\right\|+\alpha\left\|S_{2}^{\prime}(x, y)\right\| \rightarrow \inf _{S}
$$

for the function $f_{N}(x, y)$ by using quadratic or cubic splines $S(x, y)$. This problem was completely investigated in [14]. Perhaps it would be useful to recompute the amplitude distribution on the aperture $A$ after each next version of the modified reflector surface and to change the function $E(x, y)$ in the corresponding Fourier transforms $(2.2)$.

Fig. 1 shows an example of a far-field pattern of the cosecant type for the antenna with a single feed element and the reflector surface shaped by the described method. The results of the synthesized contour beam for Europe and the corresponding reflector surface form are presented in Fig. 2. Other examples can be found in [15].

\section{Conclusion}

In this work, we describe a new iterative method for numerical shaping of the locally curved shape of the reflector surface for the antenna with a single feed element. The antenna must generate a beam with a prescribed contour of its cross-section. The method has some common features with other known methods, in particular, stated in the papers from the list of references. Our method mainly differs by the technique of minimization in the problem of aperture phase synthesis, which makes it possible to shape reflectors of large diameter. The method was tested in many computing experiments and showed itself as efficient.

\section{Acknowledgments}

The authors are grateful to N.A.Baraboshkina for collaboration in the work on this paper.

\section{REFERENCES}

1. Shishlov A.V., Shitikov A.M. On the application of two-dimensional Kotelnikov's interpolation series to the synthesis of antennas with contour directional patterns // Radiophysical Signal Processing Methods. Proceedings of the Moscow Institute of Physics and Technology, 1996. P. 112-128 [in Russian].

2. Reutov A.S., Shishlov A.V. Peculiarities of step-by-step synthesis of reflectors of mirror antennas with contour directional diagrams, using a stipe representation of the mirror surface // Electromagnetic Waves and Electronic Systems, 2003. Vol. 2. P. 4-14. [in Russian]

3. Uzolin E.Yu., Kryukov I.G. Synthesis of surface profile of reflectors of single-mirror contour antennas using Zernike polynomials // Proceedings of Tomsk State University of Control Systems and Radioelectronics, 2012. Vol.2, no.2. P. 92-95. [in Russian] 

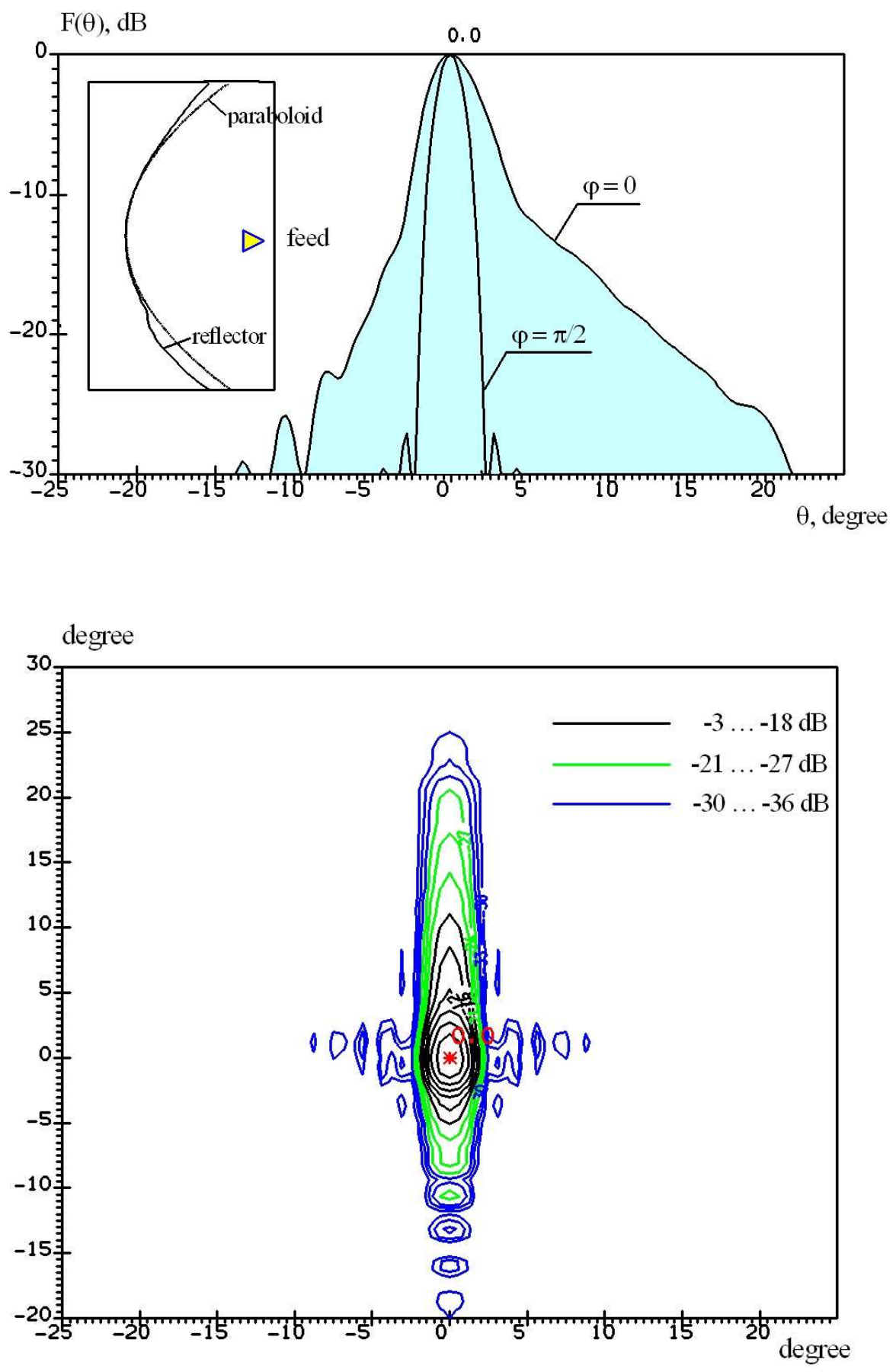

Figure 1. Example of the synthesized far-field pattern of double cosecant type 

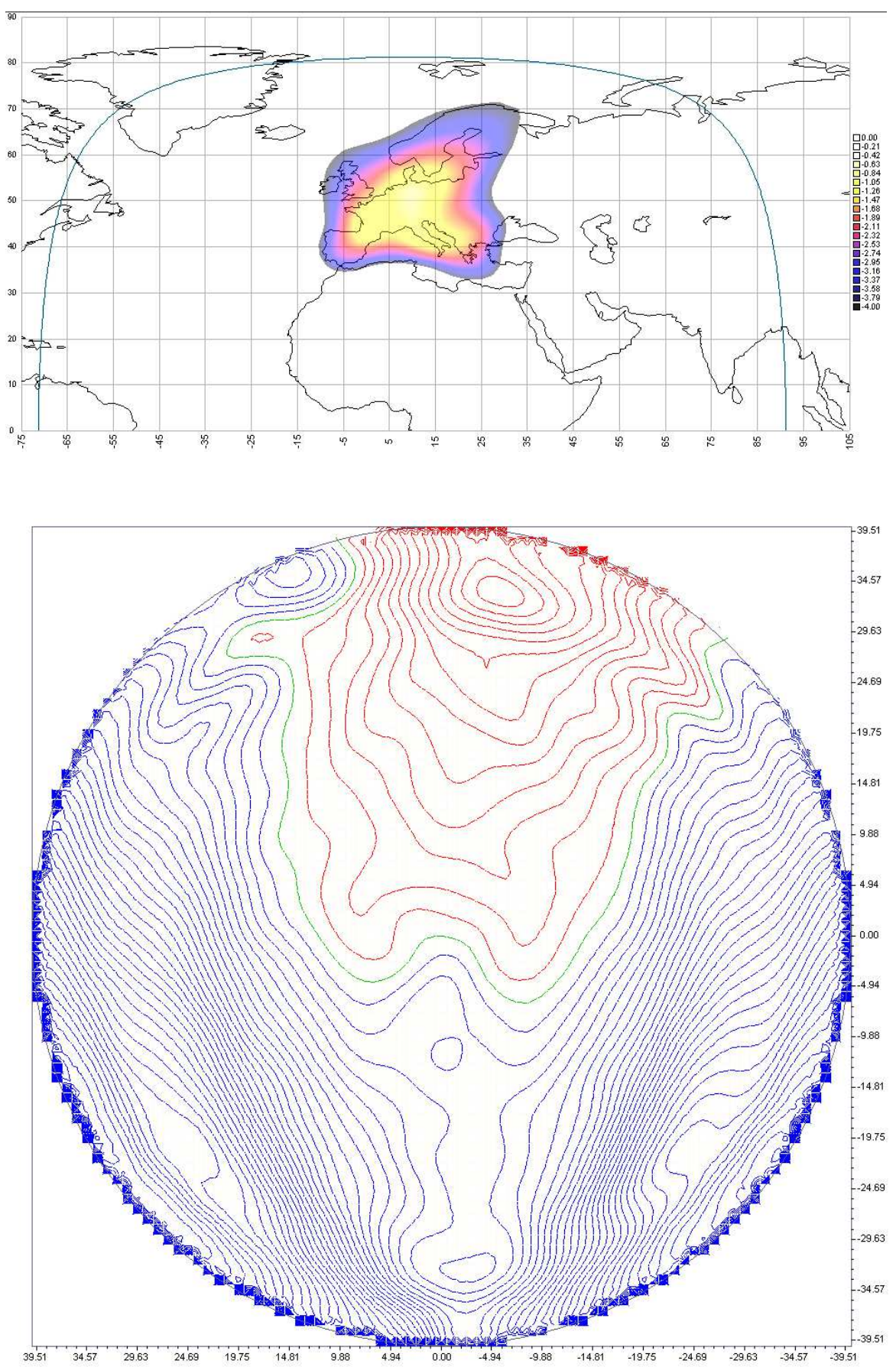

$\lambda$

Figure 2. Example of the synthesized contour beam for Europe and the corresponding reflector surface form (contour plot of a deflection of a of the synthesized reflector surface from an initial paraboloid are shown with a step 0,01 wavelength $\lambda$ ) 
4. Vipiana F., Pirinoli P., Vecchi G. Wavelet-mom analysis of 3d antennas with triangular mesh // Antennas and Propagation Society International Symposium, IEEE, 2004. P. 1471-1474. DOI: 10.1109/APS.2004.1330466

5. Woodward P.M., Lawson J.D. The theoretical precision with an arbitrary radiation-pattern may be obtained from a source of finite size // J. Institution of Electrical Engineers - Part III: Radio and Communication Engineering, IET, 1948. Vol. 95, no. 37. P. 363-370. DOI: 10.1049/ji-3-2.1948.0094

6. Jorgensen R. Coverage shaping of contoured-beam antennas by aperture field synthesis // IEE Proceedings H - Microwaves, Optics and Antennas, IET, 1980. Vol. 127, no. 4. P. 201-208. DOI: 10.1049/iph-1:19800045

7. Alan R.C., Roberto J.A. A method for producing a shaped contour radiation pattern using a single shaped reflector and a single feed // IEEE Transactions on Antennas and Propagation, 1989. Vol. 37, no. 6. P. 698-706. DOI: 10.1109/8.29356

8. Sazonov D.M., Frolov N.J. A universal method for synthesis of shaped contour beam reflector antennas with a single-element or multiple-element feeds // Proc. of the 28 Moscow Int. Conf. of Antenna theory and Technology. 1998. P. 399-402.

9. Shishlov A.V. Mirror antennas with contour directional diagrams - efficiency and limiting capabilities // Radio Engineering, 2006. Vol. 4. P. 45-50 [in Russian].

10. Bucci, DElia G., Romito G. Synthesis technique for scanning and/or reconfigurable beam reflector antennas with phase-only control // IEE. Proc.-Microw., Antennas Propag., IET, 1996. Vol. 143, no. 5 P. 402-412. DOI: 10.1049/ip-map:19960262

11. Choni Yu.I. Synthesis of antennas based on a given amplitude directional pattern // Radio engineering and electronics. 1971. Vol. 15, no. 5. P. 726-734 [in Russian].

12. Arestov V.V., Balaganskii V.S., Gusevskii V.I., Semenov B.V., Sobolev B.S., Chernyh N.I., Sharin N.P. The problem of phase synthesis DN GZA // Collection of scientific works "Radio century: Perspective ways of development of antenna systems of space communication, theory of control and pattern recognition". Ekaterinburg: UrB RAS, 1996. P. 29-55 [in Russian].

13. Sobolev B.S. Vector mathematical model of reflector antennas. // Sbornik "Raketno-kosmicheskya tehnika". Ekaterinburg: NPO avtomatiki, 2014. P. 140-151 [in Russian].

14. Sazanov A.A. Approximative properties of generalized interpolating splines in a metric linear space // Proceeding of the Steklov Institute of Mathematics, 2002. Suppl. 2. P. 155-161.

15. Semenov B.V. Effective iterative methods for contoured beams reflector antenna synthesis // European Microwave Week 2009, Conf. Proc. (EuMA 2009). P. 1500-1503. DOI: 10.23919/EUMC.2009.5296389 